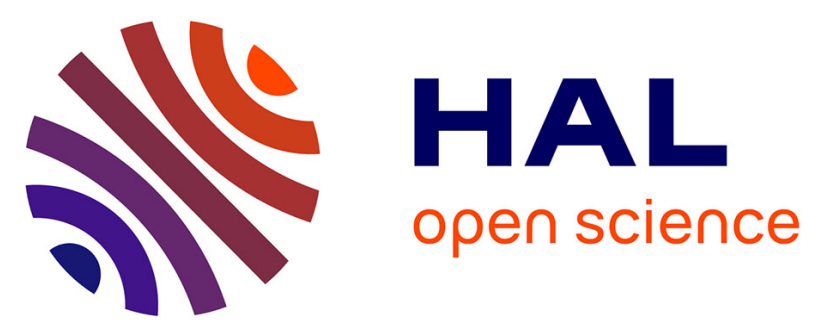

\title{
Real-time Conversion of Electrochemical Currents into Fluorescence Signals Using 8-Hydroxypyrene-1,3,6-trisulfonic Acid (HPTS) and Amplex Red as Fluorogenic Reporters
}

Christophe Demaille, Rabia A Djoumer, Arnaud Chovin, Corinne Dejous, Hamida Hallil

\section{To cite this version:}

Christophe Demaille, Rabia A Djoumer, Arnaud Chovin, Corinne Dejous, Hamida Hallil. Realtime Conversion of Electrochemical Currents into Fluorescence Signals Using 8-Hydroxypyrene-1,3,6trisulfonic Acid (HPTS) and Amplex Red as Fluorogenic Reporters. ChemElectroChem, 2021, 8 (12), pp.2298-2307. 10.1002/celc.202100517 . hal-03445077

\section{HAL Id: hal-03445077 \\ https://hal.science/hal-03445077}

Submitted on 23 Nov 2021

HAL is a multi-disciplinary open access archive for the deposit and dissemination of scientific research documents, whether they are published or not. The documents may come from teaching and research institutions in France or abroad, or from public or private research centers.
L'archive ouverte pluridisciplinaire HAL, est destinée au dépôt et à la diffusion de documents scientifiques de niveau recherche, publiés ou non, émanant des établissements d'enseignement et de recherche français ou étrangers, des laboratoires publics ou privés. 


\title{
Real-time conversion of electrochemical currents into
}

\section{fluorescence signals using 8-hydroxypyrene-1,3,6-trisulfonic acid (HPTS) and Amplex Red as fluorogenic reporters}

\author{
Rabia Djoumer, ${ }^{[a]}$ Arnaud Chovin, ${ }^{[a]}$ Christophe Demaille, ${ }^{*[a]}$ Corinne Dejous,${ }^{[b]}$ Hamida Hallil ${ }^{[b]}$
}

\author{
[a] R. Djoumer, Dr. A. Chovin, Dr. C. Demaille, \\ Laboratoire d'Electrochimie Moléculaire \\ Université de Paris \\ CNRS UMR 7591 \\ F-75006 Paris, France \\ E-mail: arnaud.chovin@u-paris.fr \\ [b] Pr. C.Dejous, Dr. H. Hallil \\ Laboratoire IMS \\ Université de Bordeaux \\ Bordeaux INP \\ CNRS UMR5218 \\ F-33405 Talence, France
}

Supporting information for this article is given via a link at the end of the document.

\begin{abstract}
This paper elaborates on an instrumental scheme for translating electrochemical reactions occurring at an electrode under potentiostatic control into fluorescent signals. We examine the performance enhancement of this device using recently introduced water-soluble fluorescent reporter systems, either the $\mathrm{pH}$-sensitive 8-hydroxypyrene-1,3,6-trisulfonic acid (HPTS) system or the electrofluorogenic Amplex Red species. The conversion of stationary and transient, cathodic and anodic electrochemical currents into fluorescence is demonstrated. We show that pure capacitive currents can also be converted to fluorescence by our setup, highlighting the rarely observed coupling between a faradaic reaction at one electrode and capacitive charging at another.
\end{abstract}

\section{Introduction}

Monitoring of electron transfer reactions by observing an electrode surface using fluorescence microscopy is the subject of intense research. ${ }^{[1-3]}$ In essence, sensitive fluorescence signal is used to reveal spatially-resolved features about surface reactivity, or to image mass transport at the electrode-solution interface. Various optical platforms have been developed enabling such fluorescence based imaging of electrode processes. ${ }^{[4-12]}$ The most "direct" experimental approach relies on using an electroactive fluorogenic species whose fluorescence emission is switched on/off upon conversion of its redox state at the electrode. ${ }^{[1,9]}$ In an alternative, "indirect" strategy the emission of a non-electroactive, but $\mathrm{pH}$-sensitive, fluorophore species can be used to transduce an electrode reaction affecting local proton concentration. ${ }^{[4,10,13]}$ Beyond mere imaging, transduction of electron transfer events into fluorescence also constitutes a very powerful amplification mechanism, since a single-electron transfer event can result in the emission of thousands of photons by the reporting fluorogenic species. Such an "optical" amplification mechanism, is in principle amenable to quantitative and time-resolved electron-to-photon transduction, and as such is worthy of interest for e.g. "nano-impact" electrochemistry. ${ }^{[14-17]}$ In this modern field of research, random collisions of electroactive entities with an ultramicroelectrode surface provoke a sequence of discrete, short (sub msec), and low magnitude (10 pA - $10 \mathrm{nA}$ ) faradaic pulses, which are typically measured by transimpedance potentiostats. ${ }^{[18,19]}$ However, it is likely that such a direct electrical readout may become impossible for studying much lower intensity pulses or for resolving short-lasting collision events, by lack of sensitivity and/or bandwidth of available current measurers. ${ }^{[20-23]}$ Hence, alternative strategies based on the transformation of the electrical signal into an easier to detect optical signal need to be developed. To this aim, various transduction platforms have been proposed, and largely reviewed, ${ }^{[2,24-26]}$ some of them are based on the conversion of a faradaic signal generated by a non-fluorogenic redox species into fluorescence emission. This conversion scheme was initially implemented using a bipolar electrode in its "closed" configuration, ${ }^{[27,28]}$ where a conductive material, through which an external driving voltage is applied, is polarized at both extremities in distinct anodic and cathodic sites to couple two electrochemical reactions. Although the applied potential is only indirectly related to the thermodynamics of the reaction of interest, the control voltage can be adjusted to initiate a "classical" (non-fluorogenic) electron-transfer reaction at one side, coupled to a simultaneous electro-fluorogenic reaction at the second pole. ${ }^{[29-32]}$ In the state of the art, this scheme has been proposed to detect impacts of single molecule on a bipolar nanoparticle by short fluorescence pulses. ${ }^{[33]}$ In a similar vein, we recently developed a fluoro-electrochemical platform enabling any electrochemical process occurring at an electrode under potentiostatic control to be convert into a spatially separated fluorescence signal. ${ }^{[34]}$ This platform, described in Scheme $1 \mathrm{a}$, relies on a classical three electrode potentiostatic setup, but where the reaction occurring at the counter-electrode can be chosen so as to produce a fluorescence species. This configuration provides an immediate and uncompromising coupling between the electrochemical reaction of interest at the working electrode and generation of fluorescence at the counter electrode. By using an epifluorescence microscope focused on the counter-electrode surface, one can therefore acquire a fluorescence signal which reports electron transfer events occurring at the working electrode. 
As a proof of principle, we previously showed that oxidation of ferrocenedimethanol could be coupled to the reduction of the fluorogenic species resazurin into resorufin, and that the resulting generation of fluorescence at the counter electrode perfectly tracked the working electrode current, provided the counter electrode was an ultramicroelectrode (UME). Using an UME was required to prevent accumulation of the fluorescent species resorufin in the vicinity of the counter electrode, thus

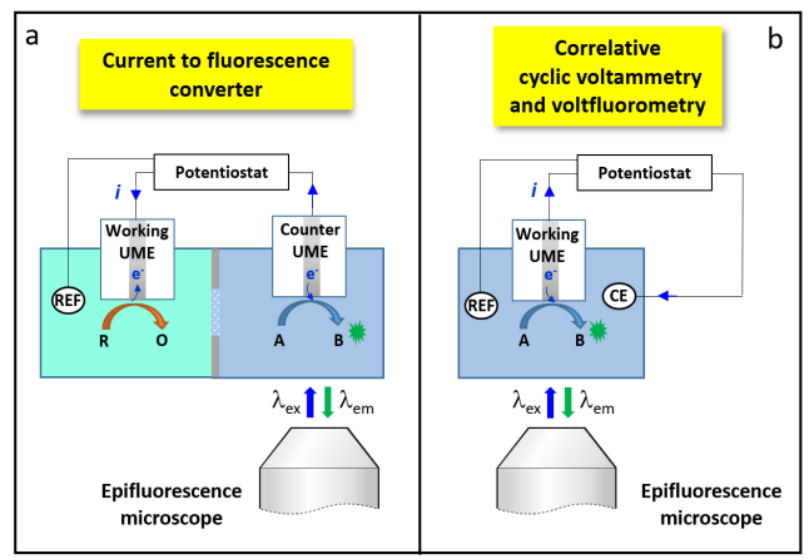

Scheme 1. (a) Working principle of the current to fluorescence converter setup. An electrochemical reaction at a working ultramicroelectrode (UME) is coupled, via a potentiostat, to a fluorogenic reaction at the counter ultramicroelectrode. The current is measured at the working electrode and fluorescence at the counter electrode. (b) Configuration used for cyclic voltammetry and voltfluorometry studies of fluorogenic systems. Both the current and fluorescent signals are simultaneously measured at the working electrode.

allowing any variation in current to be "instantaneously" translated into a correlated fluorescence variation. ${ }^{[34]}$ In the present work we intend to further investigate the performances and limitations of this faradaic current-to-fluorescence conversion scheme, and their dependence on the fluorogenic reporter used for transduction. Beyond the sensitivity criteria, we are interested in the possibility of converting short current transients into fluorescence. In this respect, we wanted to know if purely capacitive currents could equally be so-converted, an a priori provoking idea as it implies coupling capacitive charging at one electrode to a faradaic fluorogenic process at the other.

\section{Results and Discussion}

The primary aim of the present work was to assess the performances of new fluorogenic systems as reporters for our current-to-fluorescence set-up. The first system considered, recently described by Compton et al. ${ }^{[10]}$ is based on the use of the $\mathrm{pH}$ sensitive fluorophore 8-hydroxypyrene-1,3,6-trisulfonic acid (HPTS), Scheme 2A. HPTS was used to visualize the reduction of $p$-benzoquinone $(B Q)$ into $p$-hydroquinone occurring at a carbon fiber wire microelectrode. ${ }^{[10]}$ The local raise in $\mathrm{pH}$
A

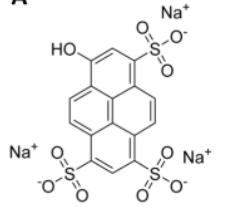

HPTS
Scheme 2. (A) Chemical structure of the fluorogenic molecule 8 hydroxypyrene-1,3,6-trisulfonic acid (HPTS). (B) Principle of $\mathrm{pH}$-induced fluorescence emission by deprotonated HPTS at an electrode where $p$ benzoquinone $(B Q)$ is reduced to $p$-hydroquinone $\left(B Q H_{2}\right)$.

due to the consumption of $\mathrm{H}^{+}$by $\mathrm{BQ}$ reduction resulted in the deprotonation of the non-fluorescent HPTS species to form its associated base, PTS (pKa 7), which is highly fluorescent, Scheme 2B.

Herein, we started by conducting coupled cyclic voltammetry and voltfluorometry studies of the HPTS/BQ system, in the configuration depicted in Scheme 1b, where the fluorescence generated at the working electrode is probed.

Cyclic voltammetry and voltfluorometry studies of the HPTS/BQ fluorogenic system

Cyclic voltammograms (CVs, current vs. electrode potential, $E$ ) and voltfluorograms (CVFs, fluorescence intensity vs. $E$ ) were recorded simultaneously at a $25 \mu \mathrm{m}$ carbon working UME, in a solution containing $1 \mathrm{mM} \mathrm{BQ}, 2 \mathrm{mM}$ HPTS in $0.1 \mathrm{M} \mathrm{KCl}$ supporting electrolyte. These data are reported in Figure 1a and Figure $1 \mathrm{~b}$, respectively. One can observe that a well-defined single $S$-shape CV wave is recorded (Figure 1a), it is characterized by a half-wave potential of $-0.18 \mathrm{~V} / \mathrm{SCE}$ and a plateau current of $5 \mathrm{nA}$, indicating a 2-electron reduction reaction. These characteristics are in agreement with the electrochemical behavior of benzoquinone in a non-buffered aqueous medium. ${ }^{[35]}$

The simultaneously recorded CVF (Figure $1 \mathrm{~b}$ ), shows that a very intense fluorescence signal is generated as the electrode is scanned cathodically, i.e. as $B Q$ is reduced. The fluorescence signal is seen to be globally similar to the CV trace, but exhibits a very large hysteresis: a more intense fluorescent signal is detected on the return vs. the forward potential scan. Interestingly we had not observed such a hysteresis previously when studying the resazurin/resorufin fluorescence reporter system. ${ }^{[34]}$
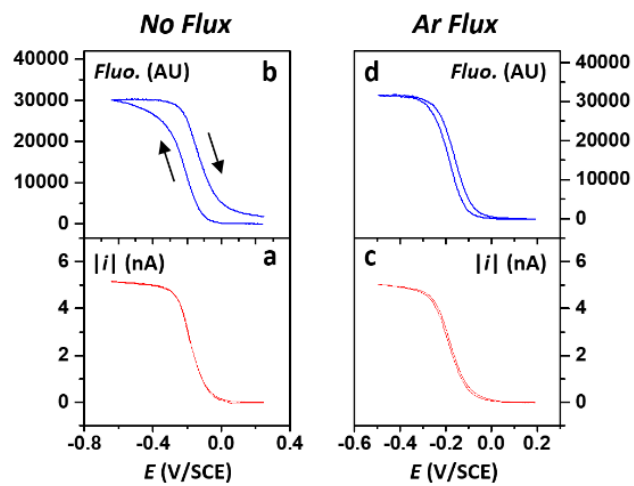

Figure 1. Cyclic voltammetry and voltfluorometry of the $\mathrm{HPTS} / \mathrm{BQ}$ fluorogenic system. (a, c) Cyclic voltammogram (CV) of $1 \mathrm{mM} \mathrm{BQ}$ in the presence of $2 \mathrm{mM}$ HPTS, at a $25 \mu \mathrm{m}$ diameter carbon UME. (b, d) Simultaneously recorded volffluorograms (CVF). Effect of convection induced by an argon flux blown over the solution: $(a, b)$ flux off, $(c, d)$ flux on. $0.1 \mathrm{M} \mathrm{KCl}$ aqueous electrolyte, scan rate $0.01 \mathrm{~V} \mathrm{~s}^{-1}$. 
After investigation we discovered that the fluorescence hysteresis obtained here could be greatly reduced, and sometimes almost totally suppressed, simply by increasing the argon flux permanently blown above the solution (to keep oxygen out of the cell), as exemplified in Figure 1d. Importantly the CV signal was unaffected by such an increase in argon flux (Figure 1a and Figure 1c). Hence, we attribute the fluorescence hysteresis to accumulation of the PTS species generated at the electrode within the field of view of the epifluorescence microscope. This result evidences that, unlike previously thought, spherical diffusion at $25 \mu \mathrm{m}$ UMEs actually needs to be complemented by a mild convection mechanism (here provided by circulating argon) in order to fully prevent accumulation of the fluorescent species in the electrode vicinity. The relative insensitivity of the stationary currents recorded at UMEs to convection, ${ }^{[36-38]}$ makes it possible, as achieved here, to set conditions where convection is strong enough to disperse the fluorescent molecules accumulating at the electrode, while not perturbing the current. Even a moderate $(\sim 100 \mathrm{~mL} / \mathrm{min})$ flux of argon gas over the surface of the $3 \mathrm{~cm} \times 1.5 \mathrm{~cm} \times 1.0 \mathrm{~cm}$ electrochemical cell was enough to generate the convective agitation required to obtain closely matching current and fluorescence signals, a prerequisite to the successful implementation of our current to fluorescence conversion setup. However, we thought relevant to search for an experimental strategy enabling to avoid accumulation of PTS $^{-}$in the vicinity of the electrode without resorting to convection. In the literature, it has been shown that such kind of accumulation could be avoided by introducing in solution a species which chemically scavenges the fluorescent molecules produced at the electrode. ${ }^{[30,39]}$ In the case of the HPTS/BQ system, Compton et al. showed that the spatial extend of the fluorescence profile developing at an electrode where $\mathrm{BQ}$ is reduced could be minimized by acidifying the medium with $\mathrm{HCl}$, since protonation of PTS into its non-fluorescent HPTS then occurred closer from the electrode surface. ${ }^{[10]}$ We thus explored this $\mathrm{pH}$-based strategy as a way of solving the "fluorescence hysteresis" problem we encountered here. Hence a series of cyclic voltammograms and voltffluorograms were recorded at a $25 \mu \mathrm{m}$ carbon working UME, in the $1 \mathrm{mM} \mathrm{BQ/2mM} \mathrm{HPTS} \mathrm{solution} \mathrm{to}$ which increasing amounts of $\mathrm{HCl}$ were added, Figure 2.

We observed that addition of even a minute amount of $\mathrm{HCl}$ (i.e. $0.05 \mathrm{mM}$ ) considerably reduced the hysteresis of the CVF signal (Figure 2d). This effect is due to PTS becoming rapidly protonated upon diffusing away from the close proximity of the electrode surface toward the now acidified bulk of the solution. Such a result validates the concept of "quenching" chemically (via protonation) the fluorescent species generated at (near) the electrode surface as a way of reducing the hysteresis of the fluorescence signal. However, if one now considers the CV signal (Figure 2c), one can see that $\mathrm{H}^{+}$addition is also

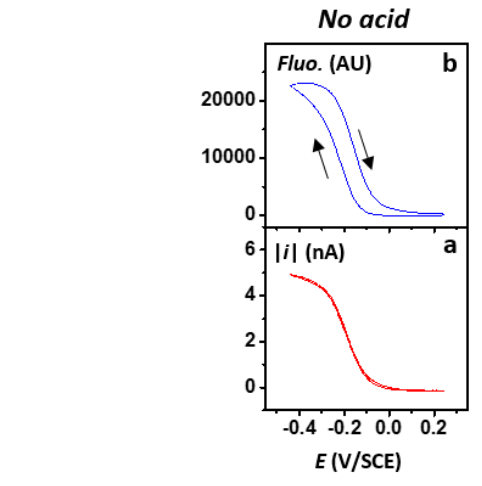

$0.05 \mathrm{mM} \mathrm{HCl}$
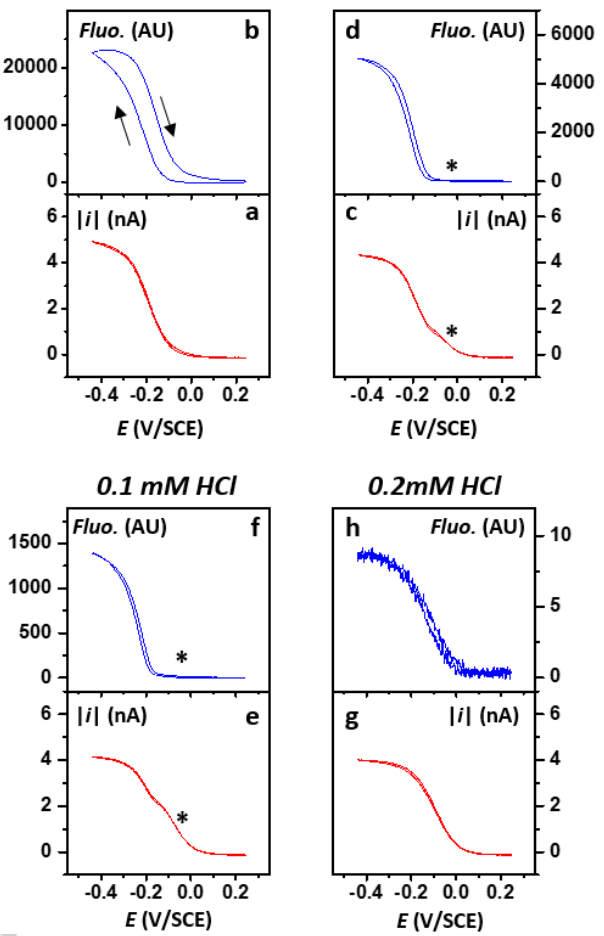

Figure 2. Cyclic voltammetry and voltfluorometry of the HPTS/BQ fluorogenic system. Effect of $\mathrm{HCl}$ addition. (a,c,e,g) Cyclic voltammogram (CV) of $1 \mathrm{mM}$ $\mathrm{BQ}$ in the presence of $2 \mathrm{mM}$ HPTS, at a $25 \mu \mathrm{m}$ diameter carbon UME. (b,d,f,h) Simultaneously recorded voltfluorograms (CVF). The concentration of added $\mathrm{HCl}$ is indicated above each CV/CVF couple. The asterisk designates the position of the pre-wave discussed in the text. $0.1 \mathrm{M} \mathrm{KCl}$ aqueous electrolyte, scan rate $0.01 \mathrm{~V} \mathrm{~s}^{-1}$

associated with the appearance of a pre-wave (designated by an asterisk), which initially gains in intensity as more $\mathrm{H}^{+}$is added (Figure 2e). The plateau current of the $\mathrm{CV}$ also decreases slightly as the concentration of $\mathrm{H}^{+}$is increased. Comparing now the CVFs presented in Figure 2(b, $d$ and $f$ ) it can be observed that the intensity of the fluorescence signal sharply decreases as the $\mathrm{H}^{+}$concentration is raised. Moreover, one can also see that almost no fluorescence is detected at the level of the prewave, whereas intense fluorescence is emitted past this wave. This poses a major problem in the perspective of using the HPTS/BQ system to transduce electrochemical current into fluorescence, which requires shape-correlated current and fluorescent signals. However, for high enough $\mathrm{HCl}$ concentration $(0.2 \mathrm{mM})$, the prewave ultimately dominates the entire current signal (Figure $2 \mathrm{~g}$ ) and the associated fluorescence signal becomes correlated in shape with the current signal (Figure 2h). The CVF then displays little hysteresis, albeit its intensity is then very low (Figure $2 \mathrm{~h}$ ). It is therefore interesting to try to understand further the CV and CVF behavior of the HPTS/BQ system in search of ways of increasing the intensity recorded in this situation. 
For this, one needs to consider in details the electron transfer and protonation steps associated with the $\mathrm{BQ}$ reduction at the electrode, and the way they affect the production of the fluorescent species PTS, on the basis of the square scheme

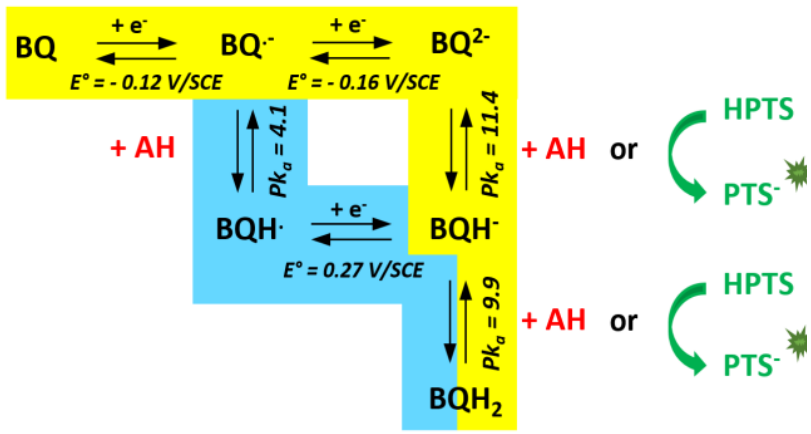

Scheme 3. Square scheme depicting the possible reduction paths for benzoquinone $(\mathrm{BQ})$ at an electrode, in the presence of an acid $(\mathrm{AH})$ and HPTS.

reproduced below and adapted from reference ${ }^{[10]}$.The above Scheme 3 is likely an oversimplification of the complex elementary electron/proton transfer processes involved, but was shown to effectively describe the behavior of the HPTS/BQ system. ${ }^{[10]}$ Besides, this reaction scheme could be easily implemented in the DigiElch simulation software enabling the following observations and conclusions to be drawn. In the absence of acid, the path highlighted in yellow is followed. BQ is reduced sequentially in two steps occurring at very close potentials, so overall a single CV wave is obtained. The dianionic species $\mathrm{BQ}^{2-}$ produced at the electrode is protonated by HPTS to yield the fluorescent product PTS. The same is true for the thus produced $\mathrm{BQH}^{-}$species, and its protonation by HPTS yields a second PTS molecule. The whole process is thus responsible for the formation of an intense fluorescent zone forming around the electrode.

In the presence of $\mathrm{H}^{+}$the path highlighted in blue is followed preferably to the yellow path (see Figure $\mathrm{S} 1$ ). The $\mathrm{H}^{+}$species protonate the intermediate species $\mathrm{BQ}^{-}$to form $\mathrm{BQH}$ which is instantaneously further reduced to form $\mathrm{BQH}^{-}$. This latter species is then protonated competitively either by $\mathrm{H}^{+}$or HPTS, protonation by $\mathrm{H}^{+}$being thermodynamically favored. Simulations revealed that the occurrence of the prewave appearing in the CVs corresponds the reduction of $B Q$ into $\mathrm{BQH}_{2}$ occurring via the blue path. This explains why almost no fluorescence signal was recorded at the level of the prewave. However, since the $\mathrm{H}^{+}$ concentration is very low compared to $\mathrm{BQ}, \mathrm{H}^{+}$is quickly depleted at the electrode surface and reduction of $B Q$ via the yellow path is restored past the prewave. Concomitantly a large fluorescent signal is then recovered, as shown in Figure $2 \mathrm{~d}$ and $2 \mathrm{f}$. The overall decrease of the intensity of the fluorescence signal with increasing $\mathrm{H}^{+}$concentration is due to $\mathrm{BQ} \cdot$ and $\mathrm{BQH}^{-}$being progressively predominantly protonated by $\mathrm{H}^{+}$rather than by HPTS.

Most interestingly, simulations also showed that by increasing the $\mathrm{H}^{+}$concentration to even a fraction of the initial $\mathrm{BQ}$ concentration it is possible to find conditions where the blue path is followed throughout the recording of the CV/CVFs. In this situation a single CV wave corresponding to the "prewave" is expected. This possibility arises from the fast diffusion of protons towards the electrode, which enable the complete reduction of $\mathrm{BQ}$ into $\mathrm{BQH}_{2}$ even though the $\mathrm{H}^{+} / \mathrm{BQ}$ concentration ratio in bulk
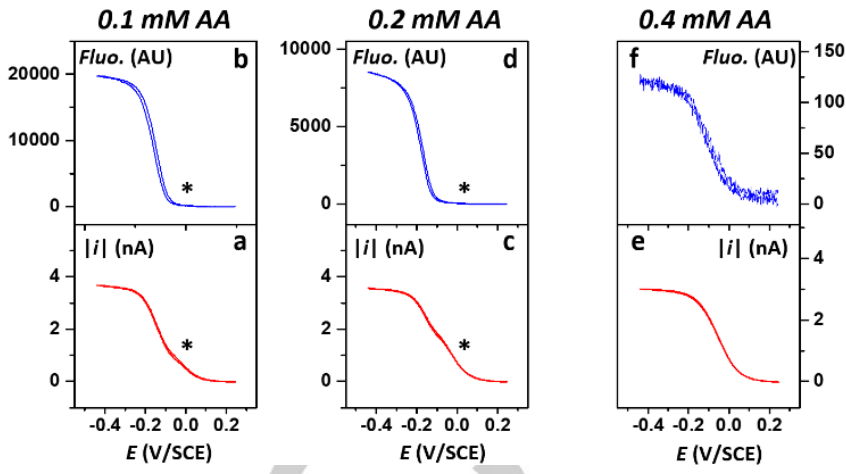

Figure 3. Cyclic voltammetry and voltfluorometry of the HPTS/BQ fluorogenic system. Effect of acetic acid (AA) addition. (a,c,e) Cyclic voltammogram (CV) of $1 \mathrm{mM} \mathrm{BQ}$ in the presence of $2 \mathrm{mM} H P T S$, and various amount of $\mathrm{AA}$, at a $25 \mu \mathrm{m}$ diameter carbon microelectrode. $(\mathrm{b}, \mathrm{d}, \mathrm{f}) \quad$ Simultaneously recorded voltfluorograms (CVF). The concentration of added AA is indicated above each CV/CVF couple. The asterisk designates the position of the pre-wave discussed in the text. $0.1 \mathrm{M} \mathrm{KCl}$ aqueous electrolyte, scan rate $0.01 \mathrm{~V} \mathrm{~s}^{-1}$

is less than unity. These conditions were obviously experimentally met here for $0.2 \mathrm{mM} \mathrm{HCl}$, as shown in Figure $2 \mathrm{~h}$. This latter value is the minimum $\mathrm{HCl}$ concentration to be used for the CV and CVF signals to display an identical shape all along the potential region explored. Increasing the $\mathrm{HCl}$ concentration further is detrimental since it simply results in decreasing further the intensity of the fluorescence signal, by making protonation of $\mathrm{BQ}^{-}$and $\mathrm{BQH}^{-}$by $\mathrm{H}^{+}$, rather than by HPTS, even more favorable.

Hence one could thus say we did find the appropriate conditions for the HPTS/BQ system to be used as a basis of our current-tofluorescence conversion scheme. However, by comparing Figure $2 \mathrm{~d}$ and $2 \mathrm{f}$ to Figure $2 \mathrm{~h}$, one can see that finding these conditions was made at the expense of the fluorescence intensity which dropped by two orders of magnitude as $\mathrm{HCl}$ was added.

One thus can conclude that even though adding $\mathrm{HCl}$ to the HPTS/BQ system may be a good strategy for increasing the resolution in electrochemical fluorescence microscopy, as originally aimed at by Compton et al., ${ }^{[10]}$ or like here for minimizing the hysteresis of the fluorescence signal, we show here that the associated large loss of fluorescence intensity limits its usability. This is due both to $\mathrm{H}^{+}$inducing a change in reaction path along the square scheme and also competing favorably with $\mathrm{HPTS}$ for the reduction of $\mathrm{BQ}^{2-}$ and $\mathrm{BQH}^{-}$species. In an attempt to alleviate this problem, we tried to replace $\mathrm{H}^{+}$by a weak acid, acetic acid $(A A, p K a=4.75)$, and recorded CVs and CVFs at the carbon UME in the $1 \mathrm{mM} / 2 \mathrm{mM} \mathrm{HPTS/BQ}$ solution for increasing concentrations of this acid, Figure 3.

We observed that adding acetic acid was effective in drastically decreasing the hysteresis of the CVF wave (compare Figure $2 b$ and Figure $3 \mathrm{~b}$ ). However, addition of acetic acid to the solution also resulted in the appearance of the same prewave we observed above when $\mathrm{HCl}$ was used to acidify the solution. This indicates that protonation of $\mathrm{BQ}^{-}$by acetic acid occurred to a significant extend, in spite of the fact that $\mathrm{BQH}$ is expected to be a slightly stronger acid than $\mathrm{AA}$ (estimated $\mathrm{pKa}\left(\mathrm{BQH} / \mathrm{BQ}^{-}\right)=$ 4.1 vs. 4.75 for $A A)$. Nevertheless, by increasing the $A A$ concentration conditions could be found where the "prewave" dominated the CV signal which was then shape-correlated with the CVF signal (Figure 3e and 3f). Interestingly, the fluorescence intensity which was then recorded was $\sim 10$ times higher than 
when $\mathrm{HCl}$ was used to acidify the solution. This is obviously a benefit of $\mathrm{AA}$ competing with HPTS for the protonation of $\mathrm{BQ}^{2-}$ and $\mathrm{BQH}^{-}$less favorably than $\mathrm{H}^{+}$.

Hence, we have demonstrated that indirect ( $\mathrm{pH}$-induced) emission of fluorescence triggered by a proton consuming faradaic reaction at an UME can result in fluorescence signals closely tracking the current generation. More precisely, for the HPTS/BQ system, we identified the experimental conditions to ensure simultaneously the generation of a single CV wave associated to an intense enough, hysteresis-free and shapecorrelated fluorescence signal, Figure $(3 e, f)$.

We next examined the possibility of using this finely tuned system for reporting a generic oxidation reaction in our currentto-fluorescence conversion scheme.

\section{Converting a generic oxidative electrochemical reaction into fluorescence using the optimized HPTS/BQ/AA system as a reporter}

To do so the counter electrode compartment of the set-up described in Scheme 1a was filled with a $0.1 \mathrm{M} \mathrm{KCl}$ aqueous solution containing $1 \mathrm{mM}$ HPTS, $2 \mathrm{mM} \mathrm{BQ}, 0.4 \mathrm{mM} \mathrm{AA}$. The solution was degassed with argon, but, importantly, the gas flow over the compartment was interrupted during measurements. A $25 \mu \mathrm{m}$ carbon UME was used as a counter electrode. The working electrode compartment contained $1 \mathrm{mM}$ ferrocene dimethanol $(\mathrm{Fc})$ dissolved in a $0.1 \mathrm{M} \mathrm{NaClO}_{4}$ aqueous electrolyte. The working electrode was a $25 \mu \mathrm{m}$ diameter platinum UME.

The cyclic voltammogram recorded at the working electrode, and the fluorescence intensity simultaneously measured at the counter electrode, are plotted as a function of the working electrode potential in Figure $4 \mathrm{a}$ and $4 \mathrm{~b}$, respectively.
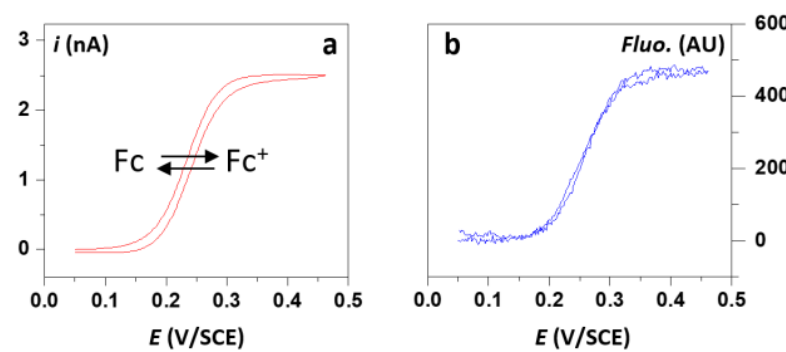

Figure 4. Converting an anodic faradaic current into a fluorescence signal, using the optimized HPTS/BQ/AA system as a reporter. Oxidation of ferrocene dimethanol $(\mathrm{Fc})$ at the working electrode under potentiostatic control, was coupled to fluorescence emission due to benzoquinone reduction in the presence of HPTS and acetic acid, at the counter electrode. (a) Cyclic voltammogram of ferrocenedimethanol at the $25 \mu \mathrm{m}$ platinum working ultramicroelectrode. (b) Fluorescence signal simultaneously recorded at a $25 \mu \mathrm{m}$ diameter carbon counter ultramicroelectrode. The working and counter electrode compartments contained respectively $1 \mathrm{mM} \mathrm{Fc}$ in a $0.1 \mathrm{M} \mathrm{NaClO}_{4}$ solution, and $1 \mathrm{mM}$ benzoquinone, $2 \mathrm{mM}$ HPTS, $0.4 \mathrm{mM}$ acetic acid in aqueous $0.1 \mathrm{M} \mathrm{KCl}$. Scan rate $0.01 \mathrm{~V} \mathrm{~s}^{-1}$

It can be seen that the resulting voltammogram corresponding to the oxidation of ferrocenedimethanol, and the CVF generated by the HPTS/BQ reporter couple at the counter electrode, are very similar in shape. This result demonstrates that the system, once optimized, can be used as an efficient fluorogenic reporter in our set-up, enabling the oxidation current generated at an electrode under potentiostatic control to be converted into a hysteresisfree fluorescence emission. Compared to the resazurin/resorufin reporter system used previously, the HPTS/BQ/AA system eliminates the fluorescence hysteresis problem without the need to resort to added convection. This of course enables closer tracking of the current by the fluorescent signal. Moreover, by avoiding accumulation of fluorescent species in the vicinity of the electrode, this new system is also expected to yield a better time resolution (i.e. bandwidth) for the current-to-fluorescent set-up.

In order to explore this possibility, we conducted chronoamperometry measurements, where the potential of the working electrode was pulsed from 0.05 to $0.45 \mathrm{~V} / \mathrm{SCE}$ and back, so as to trigger on, then off, the oxidation of ferrocenedimethanol at the working electrode. The current and fluorescent signals, generated at the working and counter UMEs respectively, were acquired simultaneously. Figure 5 presents the corresponding current and fluorescence time trace signals recorded when a series of such short $(0.1 \mathrm{~s}$ duration) potential pulses were applied to the working electrode, with the HPTS/BQ reporting system being used either non-optimized (i.e. with no AA added, left panel), or optimized by the addition of $0.4 \mathrm{mM} \mathrm{AA}$ (right panel).

As can be seen, in both cases, the potential steps shown in Figure $5 a$ and $5 a^{\prime}$ resulted in similar current transients (Figure $5 \mathrm{~b}$ and $5 b^{\prime}$ ), indicating that the oxidation of Fc at the working electrode was alternatively turned on and off. Examining Figure $5 c$ and $5 c^{\prime}$, it is seen that intense fluorescent transients, well correlated with the current transients were then recorded, both in the presence and absence of AA. However, one can also notice that the fluorescent transients recorded in the absence of acid are characterized by a sharply rising portion followed by a much more slowly decaying part. This tailing part extends over a time region where the potential has been brought back to $0.05 \mathrm{~V}$,

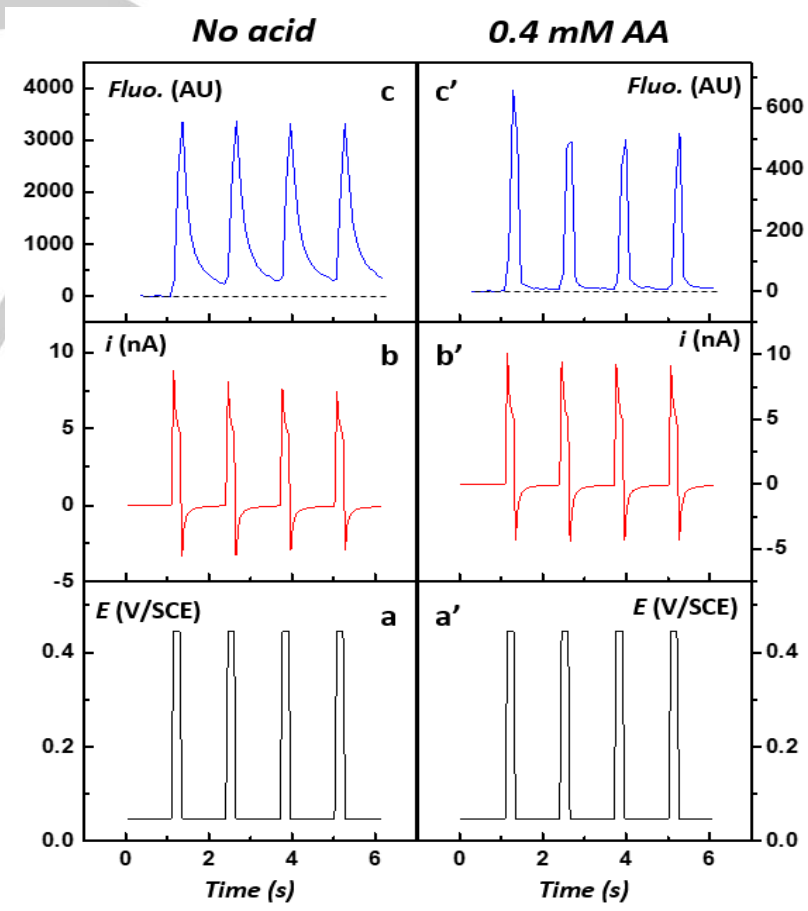

Figure 5. Converting transient anodic faradaic currents into fluorescence signals, using the optimized HPTS/BQ system as a reporter. Evidencing gain in bandwidth by acetic acid addition. Left $(a, b, c)$ - No acid added. Right (a', b', c') in the presence of $0.4 \mathrm{mM}$ acetic acid (AA). (a,a') Series of four short ( $0.1 \mathrm{~s}$ duration) potential pulses applied to a $25 \mu \mathrm{m}$ platinum working microelectrode immersed in a ferrocenedimethanol containing solution. (b,b') Resulting current transients. (c,c') Corresponding fluorescence emission transients recorded at the counter microelectrode, immersed in a HPTS/BQ containing solution. The working and counter electrode compartments contained respectively $1 \mathrm{mM} \mathrm{Fc}$ in a $0.1 \mathrm{M}$ $\mathrm{NaClO}_{4}$ solution, and $1 \mathrm{mM}$ Benzoquinone, $2 \mathrm{mM}$ HPTS in aqueous $0.1 \mathrm{M}$ $\mathrm{KCl}$. The counter electrode was a $25 \mu \mathrm{m}$ carbon UME. 
where oxidation of ferrocenedimethanol has ceased and current is zero.

Besides, it can also be observed that, because of the diffusive tail, the fluorescent signal does not drop to zero in the time frame of $1.5 \mathrm{~s}$ separating successive pulses. Such a behavior is a clear demonstration of the accumulation of PTS species in the vicinity of the counter electrode, which diffuses away only slowly. Turning now to the data acquired in the presence of $A A$ one can observe that the fluorescent transients do not display the tailing region obtained in the absence of $A A$, but at the opposite return rapidly to zero when the working electrode is set back to $0.05 \mathrm{~V}$, i.e. when the oxidation of ferrocenedimethanol is switched off. This shows that the optimized HPTS/BQ/AA system enables the conversion of a current to a fluorescence signal at time scale of at least $0.1 \mathrm{~s}$. This result is important as it opens the possibility of detecting short lived events generating low faradic currents into easier to detect fluorescence signals. Indeed, due to the much higher sensitivity, and potentially much better bandwidth, of light detection as compared to current measurement, we expect that our setup could for example be used to resolve the collision of nanoparticles with electrode surfaces. Before this goal is reached significant increase in bandwidth and current-tofluorescence conversion sensibility will have to be demonstrated. Converting a generic reductive electrochemical reaction into fluorescence using Amplex Red system as a reporter

In our initially reported work, ${ }^{[34]}$ and so far here, we have shown that generic oxidative reactions could be transduced into fluorescence signals. Yet the question arose if reductive reactions could be equally transduced. This of course requires selecting a suitable oxidizable fluorogenic compound to be introduced in the counter electrode compartment. Following Bouffier et al., ${ }^{[40]}$ we selected Amplex Red, a non-fluorescent species which can be irreversible oxidized at an electrode to form the highly fluorescent resorufin species (scheme 4).

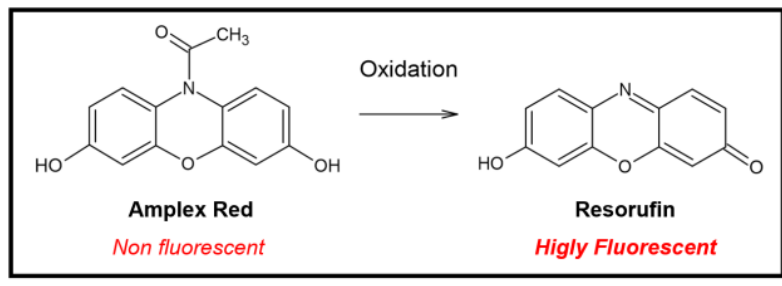

Scheme 4. Global scheme for the fluorogenic oxidation of the non fluorescent Amplex Red to the fluorescent resorufin species.

We first conducted cyclic voltammetry and voltfluorometry studies of the oxidation of Amplex Red $(0.25 \mathrm{mM}$ in pH7.4 PBS buffer) at a $25 \mu \mathrm{m}$ platinum ultramicroelectrode. The
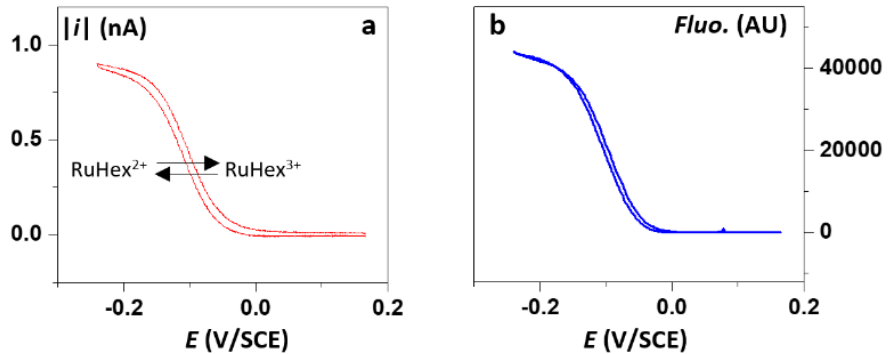

Figure 6. Converting a cathodic faradaic current into a fluorescence signal. The reduction of hexaammineruthenium(III) (RuHex), ) at the working electrode under potentiostatic control, was coupled to fluorescence emission due to Amplex Red oxidation at the counter electrode. (a) Cyclic voltammogram of RuHex at the $25 \mu \mathrm{m}$ gold working ultramicroelectrode. (b) Fluorescence signal simultaneously recorded at a $25 \mu \mathrm{m}$ diameter platinum counter ultramicroelectrode. The working and counter electrode compartments contained respectively $0.5 \mathrm{mM}$ RuHex in a $0.1 \mathrm{M} \mathrm{KCl}$ aqueous solution, and $0.25 \mathrm{mM}$ Amplex Red in $0.1 \mathrm{M}$ PBS buffer $\mathrm{pH} 7.4$. Scan rate $0.01 \mathrm{~V} \mathrm{~s}^{-1}$. simultaneously acquired CV and CVF showed that oxidation of Amplex Red at the UME started around 0.35 V/SCE and was associated with a strong emission of fluorescence, due to the production of resorufin, Figure S2. ${ }^{[40]}$ Importantly the CV and CVF signals were closely correlated in shape. We thus placed Amplex Red (0.25 mM in pH 7.4 PBS buffer) in the counter electrode compartment of our setup and hexaammineruthenium(III) (RuHex), a model reducible compound, in the working compartment. The working and counter electrodes were $25 \mu \mathrm{m}$ diameter gold and platinum microelectrodes, respectively. The working electrode potential was then scanned negatively, from $+0.15 \mathrm{~V} / \mathrm{SCE}$ to $-0.24 \mathrm{~V} / \mathrm{SCE}$ and back. The simultaneously recorded $\mathrm{CV}$ at the working electrode, and CVF at the counter electrode are presented in Figure $6 \mathrm{a}$ and $6 \mathrm{~b}$, respectively.

One can see that the CV current, corresponding to the oneelectron reduction of RuHex at the working electrode, is associated with the generation of a very intense fluorescence signal at the counter electrode. A very good similarity in shape is also observed between the current and fluoresce signals, demonstrating the possibility of converting a reductive faradaic current due to the reduction of a "generic" species at a working UME into a fluorescence signal. Please note that, to obtain such a hysteresis-free fluorescence signal, a high enough flux of argon had to be maintained over the counter electrode compartment during the measurements, so as to avoid accumulation of resorufin. Maintaining this flux was also required as Amplex Red tends to spontaneously photo-oxidize under illumination in the presence of even traces of oxygen in solution. ${ }^{[41]}$ However, the main advantage of using Amplex Red as a reporter in our setup is the very high intensity of the generated fluorescence. Indeed, on the basis of Figure 6 one sees that a $1 \mathrm{nA}$ current is converted into a fluorescence intensity of 40000 arbitrary unit (AU). Considering that the nonoptimized setup we are currently using enables the reliable detection of $10 \mathrm{AUs}$, one can immediately envision that currents in the order of $1 \mathrm{pA}$ could be readily transduced into easily detectable fluorescence signals using Amplex Red as a reporter.

\section{Can a capacitive current be also converted into fluorescence?}

In view of recording current and associated fluorescent transients, we wondered if a purely capacitive current would be converted into a fluorescence signal by our setup, just like faradic currents readily are. Since our setup relies on the fact that a faradic fluorogenic reaction occurs at the counter electrode, this comes down to the question of whether such a faradaic reaction can be coupled to a capacitive charging process at the working electrode. The difference in nature of these two processes, and notably the fact that in the faradaic case an electron is exchanged between a soluble species and the electrode, whereas in the capacitive case no such transfer occurs, makes this a provocative question. To settle it experimentally, we used a conductive silicon surface coated by a $200 \mathrm{~nm}$ thick insulating $\mathrm{SiO}_{2}$ layer as the working electrode of our setup. At such an electrode, faradaic electron transfer is totally blocked. Besides, the working electrode compartment contained solely the electrolyte $\left(0,1 \mathrm{M} \mathrm{NaClO}_{4}\right)$. A $25 \mu \mathrm{m}$ carbon UME was used as a counter electrode, it was immersed into a $0.25 \mathrm{mM}$ Amplex Red solution in $0.1 \mathrm{M}$ PBS buffer $\mathrm{pH} 7.4$ 

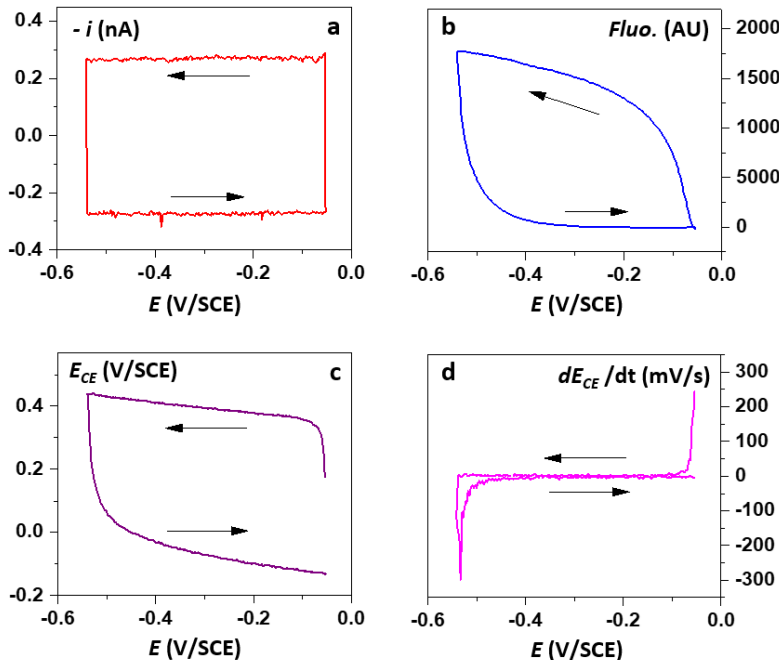

Figure 7. Converting a capacitive current into a fluorescence signal. (a) Purely capacitive cyclic voltammogram (CV) recorded at a $\mathrm{Si} / \mathrm{SiO}_{2}$ working electrode $\left(2 \mathrm{~cm}^{2}\right.$ area, capacitance, $\left.\mathrm{C}=30 \mathrm{nF}\right)$ in $0.1 \mathrm{M} \mathrm{NaClO}_{4}$ aqueous electrolyte. (b) Corresponding fluorescent signal simultaneously recorded at the $25 \mu \mathrm{m}$ diameter counter electrode $(C=0,1 \mathrm{nF})$ immersed in an Amplex Red solution. (c) Variation of the potential of the counter electrode $\left(E_{C E}\right)$ measured during the CV. (d) Time derivative of the counter electrode potential. The counter electrode compartment contained $0.25 \mathrm{mM}$ Amplex Red in 0.1 M PBS buffer $\mathrm{pH}$ 7.4. Scan rate

(Figure 7). The capacitance of the WE and CE were of $30 \mathrm{nF}$ and $\sim 0.1 \mathrm{nF}$, respectively. Such an asymmetry insured that generation of a capacitive current at the WE could not be simply counter-balanced by charging the $\mathrm{CE}$, but would necessarily involve a faradaic reaction. Upon scanning the potential of the working electrode, at $10 \mathrm{mV} / \mathrm{s}$, the $\mathrm{CV}$ shown in Figure $7 \mathrm{a}$ was obtained. The corresponding fluorescence signal, simultaneously recorded at the counter UME, is displayed in Figure $7 \mathrm{~b}$. Also shown is the potential of the counter electrode, $E_{C E}$, Figure $7 \mathrm{c}$, and its time derivative, Figure $7 \mathrm{~d}$. As seen, the CV signal is perfectly rectangular, indicating that, because of the thick $\mathrm{SiO}_{2}$ coating covering the working electrode, a purely capacitive current is generated at this electrode. Most interestingly, comparing Figure $7 \mathrm{a}$ and $7 \mathrm{~b}$ reveals that, when the working electrode potential was scanned cathodically, an intense fluorescence signal was generated at the counter electrode. At the opposite, upon reversal of the potential scan direction, the fluorescence signal quickly decayed to zero. Such a behavior indicates that during the cathodic scan at least part of the charging current of the working electrode was counterbalanced by the anodic current due to oxidation of Amplex Red at the counter electrode. During the return scan the capacitive current of the working electrode became anodic, and thus could not be compensated for by the oxidation of Amplex Red, which thus immediately ceased, hence the resulting fluorescence decay. Such a scenario is confirmed by examining the variation of the counter electrode potential recorded during the $\mathrm{CV}$, Figure $7 \mathrm{c}$ and $7 \mathrm{~d}$. One can observe that the beginning of the CV scan, the counter electrode potential increases rapidly, at an instantaneous scan rate $\mathrm{d} E_{C E} / \mathrm{dt}$ in the order of a few hundreds of millivolts per seconds. This abrupt change in $E_{C E}$ generates an anodic capacitive current in the counter electrode which exactly counterbalances the initial charging current of the working electrode. However, when $E_{C E}$ reaches a potential of $0.36 \mathrm{~V} / \mathrm{SCE}$ corresponding to the onset of Amplex Red oxidation (Figure S2), the potential of the counter electrode almost stabilizes, only varying very slowly, at about $2 \mathrm{mV} / \mathrm{s}$. Figure 7 also reveals that, upon reversal of the scan direction, the potential of the counter electrode varied initially rapidly, to generate enough capacitive current to counterbalance the working electrode current, and then much more slowly when $E_{C E}$ approached $-0.1 \mathrm{~V} / \mathrm{SCE}$, indicating that at this potential some non-defined reduction reaction occurs at the counter electrode. The above results demonstrate that a purely capacitive working electrode current can indeed be counterbalanced by a faradaic current at the counter electrode. This coupling between a faradaic reaction occurring at an electrode with a capacitive charging at another has been rarely observed directly. We are actually aware of only one report of a similar experimental observation, by Bard et al., ${ }^{[42]}$ who observed the occurrence of an electrochemiluminescent faradaic reaction at a working electrode coupled with charging of a capacitive counter electrode. This "capacitive coupling" phenomenon has since been shown to have far reaching consequences, such as enabling single molecule cycling between two electrodes forming a closed-gap, ${ }^{[43]}$ and is expected to be ubiquitous in single molecule nano-electrochemistry. ${ }^{[44]}$ Of particular interest here, we have shown that capacitive currents can indeed be transduced into fluorescence signals by our conversions setup. However, there is then no perfect shape identity between the current and fluorescence signals (compare Figure $7 \mathrm{a}$ and $7 \mathrm{~b}$ ). This is due to the fact that, upon adjusting the counter electrode potential, the potentiostat actually generates a capacitive current that can be enough to counter balance part of the current flowing through the working electrode. Yet, the counter electrode potential eventually becomes such that the faradaic fluorogenic reaction is ultimately triggered. In the general case, capacitive currents can thus contribute to fluorescence generation, in parallel to faradaic currents, but to an extend that depends on the exact details of the experimental system examined. For example, for the current transients presented in Figure 5, we evidenced that capacitive contribution existed, but was essentially negligible (see Figure S3).

\section{Conclusion}

Herein we confirmed that transduction of a cathodic faradaic current at an electrode under potentiostatic control could be quantitatively coupled with the emission of fluorescence at the counter electrode by a fluorogenic reporter species, using a simple setup we proposed earlier. We experimentally addressed some of the limits of this setup and extended its possibilities. We notably identified accumulation of the fluorescence species produced at the microelectrode in the field of view of the epifluorescence microscope as an interfering phenomenon, notably limiting the bandwidth of the current to fluorescent conversion process. We showed that such accumulation could be largely and simply avoided by mild convective agitation of the solution, for example by flushing the cell with a modest flux of inert gas.

We also demonstrated the possibility of using an indirect, $\mathrm{pH}$ modulated fluorescent reporter system in our conversion scheme, namely the HPTS/BQ system. We showed that this system can be rendered intrinsically immune to the fluorescence accumulation problem via the addition of an acid into the 
HPTS/BQ solution. However, we also evidenced that, addition of acid can induce both a detrimental change in the $B Q$ reduction pathway and a loss of fluorescence intensity. Nevertheless, it was shown that by using acetic acid to acidify the solution, optimal conditions could be found where conversion to fluorescent of both steady state and transient faradaic currents could be achieved.

The use of our converter was extended to the conversion of anodic faradaic currents into fluorescence, by making use of Amplex Red as the fluorescence reporter. We observed that this system generated a particularly intense fluorescence signal, enabling an (indicative) conversion sensitivity factor of $40000 \mathrm{AU}$ per nA.

Finally, we addressed the question of whether a purely capacitive current could be converted into a fluorescent signal as well. We showed that this was effectively the case, and in doing so observed a seldom reported case of coupling between a faradaic reaction at an electrode with a purely capacitive process at another.

\section{Experimental Section}

Chemicals and electrolytic solutions: p-benzoquinone BQ $(99,5 \%), 8$ hydroxypyrene-1,3,6-trisulfonic acid trisodium salt HPTS (96\%), Amplex Red AR (98 \%), 1-1'-ferrocenedimethanol (97\%) and hexaammineruthenium(III) chloride $(98 \%)$ were purchased from SigmaAldrich and used as received. Diluted solutions of chlorohydric acid $(\mathrm{HCl})$ and acetic acid (AA) were prepared from commercial $1 \mathrm{M}$ stock solutions (TitriNorm VWR Chemicals). All aqueous electrolytes containing $0.1 \mathrm{M}$ $\mathrm{KCl}, 0.1 \mathrm{M} \mathrm{NaClO}_{4}$ or $0.1 \mathrm{M}$ PBS pH7.4 buffer were made using doubledeionized water (Milli-Q Millipore $18.2 \mathrm{M} \Omega \mathrm{cm}^{-1}$ resistivity). All solutions were freshly prepared and deoxygenated with Argon gas for 20 minutes before experiments and between measurements. A blanket of argon was maintained above the solution during the measurements, unless otherwise stated in the text.

Electrodes: $25 \mu \mathrm{m}$ diameter platinum, gold and carbon disc ultramicroelectrodes were all hand-made items. The $\mathrm{Si} / \mathrm{SiO}_{2}$ electrode was a silicon surface coated by a $200 \mathrm{~nm}$ thick insulating $\mathrm{SiO}_{2}$ (Ted Pella Inc.), its surface area was $2 \mathrm{~cm}^{2}$. Dri-Ref electrode (Dri-Ref-2SH, WPI) was used as reference electrode. All reported potentials are referenced vs. saturated calomel electrode (SCE) potential (+ $0.05 \mathrm{~V}$ vs. Dri-Ref).

Electrochemical epifluorescence platform Fluorescence imaging of electrode surfaces was carried out using a far-field inverted microscope (IX71, Olympus) interfaced with a home-built low-current potentiostat, and placed in a Faraday cage. A specially designed LABVIEW software was used to synchronize both acquisitions of electrochemical current (CV or chronoamperometry) and fluorescence. All cyclic measurements were performed with an acquisition time of $200 \mathrm{msec}$ for both current and fluorescence signals. Transient measurements were recorded with an acquisition time of $50 \mathrm{msec}$ for current and $100 \mathrm{msec}$ for fluorescence. To ease visual comparison between signals, forward CV waves (either anodic or cathodic) are displayed as positive values. The microelectrode to observe was immersed in one of the two separated compartments of a custom-made glass cell containing $\sim 4 \mathrm{~mL}$ of solution of specified fluorogenic system, AR or HPTS/BQ. The liquid cell had a flat transparent window as the bottom to enable the visualization of the microelectrode surface of interest (working or counter electrode, according to the configuration to consider - see Scheme 1 for details) in the field of view of a long-working distance objective 40X $0.55 \mathrm{NA}$ (Olympus SLCPIFI 40X/0.55 Ph2). A built-in additional 1.6X magnification lens was systematically inserted. The electrode was precisely positioned in the focal plane of the objective with a 3D micromanipulator (Sutter Instrument) under bright-field illumination, at a distance of $\sim 500 \mu \mathrm{m}$ from the glass optical window. Excitation light was provided by a $100 \mathrm{~W}$ halogen lamp through an epifluorescence mirror unit. "Green" excitation U-MWG2 unit (Olympus) was used for Amplex Red/resorufin fluorogenic system (bandpass excitation filter at $530 \pm 20 \mathrm{~nm}, 570 \mathrm{~nm}$ dichroic mirror, long pass $590 \mathrm{~nm}$ emission filter), while "blue" U-MWB2 unit (Olympus) was chosen for HPTS fluorescence (bandpass excitation filter at $475 \pm$ $15 \mathrm{~nm}, 500 \mathrm{~nm}$ dichroic beam splitter and long pass $520 \mathrm{~nm}$ emission filter). Fluorescence intensity was collected onto a back-illuminated CMOS camera (KURO 1200B, Princeton Instruments, 95\% QE, pixel size $11 \times 11 \mu \mathrm{m}$ ), which records videos with $50-200 \mathrm{msec}$ exposure time per image, simultaneously with the electrochemical acquisition. Variation of fluorescence intensity over time was measured from a sequence of images of the same region of interest (ROI) of $100 \times 100$ pixels ( $20 \times 20$ $\mu \mathrm{m}$ in real scale) encompassing the electroactive area of the microelectrode. Mean background intensity acquired on the first image of each video was subtracted from subsequent images to derive the voltfluorogram (CVF) profiles. Camera control and image subtraction were performed with the custom made LABVIEW software, through the connected LightField 6.0 application (Princeton instruments). No further image processing was carried out.

Simulations. CVs of BQ at micro-sized electrodes, in the presence of HPTS, with/without added acid, were simulated using the DigiElch professional V7 software.

\section{Acknowledgements}

This study was supported by the IdEx Université de Paris, ANR-18-IDEX0001 ("Action Emergence en Recherche 2019 »).

\section{Keywords: Electrochemistry • Fluorescence • Low current measurement $•$ Capacitive coupling}

[1] L. Bouffier, T. Doneux, Curr. Opin. Electrochem. 2017, 6, 31-37.

[2] R. Hao, Z. Peng, B. Zhang, ACS Omega 2020, 5, 89-97.

[3] D. Bizzotto, Curr. Opin. Electrochem. 2018, 7, 161-171.

[4] R. C. Engstrom, S. Ghaffari, H. Qu, Anal. Chem. 1992, 64, 25252529 .

[5] S. Cannan, I. Douglas Macklam, P. R. Unwin, Electrochem. commun. 2002, 4, 886-892.

[6] N. C. Rudd, S. Cannan, E. Bitziou, I. Ciani, A. L. Whitworth, P. R. Unwin, Anal. Chem. 2005, 77, 6205-6217.

[7] C. Amatore, A. Chovin, P. Garrigue, L. Servant, N. Sojic, S. Szunerits, L. Thouin, Anal. Chem. 2004, 76, 7202-7210.

[8] C. Renault, K. Marchuk, H. S. Ahn, E. J. Titus, J. Kim, K. A. Willets, A. J. Bard, Anal. Chem. 2015, 87, 5730-5737.

[9] T. Doneux, L. Bouffier, B. Goudeau, S. Arbault, Anal. Chem. 2016, 88, 6292-6300.

[10] M. Yang, C. Batchelor-Mcauley, E. Kätelhön, R. G. Compton, Anal. Chem. 2017, 89, 6870-6877.

[11] A. De Poulpiquet, B. Goudeau, P. Garrigue, N. Sojic, S. Arbault, T. Doneux, L. Bouffier, Chem. Sci. 2018, 9, 6622-6628.

[12] J. Lu, Y. Fan, M. D. Howard, J. C. Vaughan, B. Zhang, J. Am. Chem Soc. 2017, 139, 2964-2971.

[13] L. Bouffier, T. Doneux, B. Goudeau, A. Kuhn, Anal. Chem. 2014, 86, 3708-3711.

[14] K. Mathwig, T. J. Aartsma, G. W. Canters, S. G. Lemay, Annu. Rev. Anal. Chem. 2014, 7, 383-404.

[15] T. Albrecht, J. MacPherson, O. Magnussen, D. Fermin, R. Crooks, J Gooding, T. Hersbach, F. Kanoufi, W. Schuhmann, C. Bentley, N. Tao, S. Mitra, K. Krischer, K. Tschulik, S. Faez, W. Nogala, P. 
Unwin, Y. Long, M. Koper, Z. Tian, M. A. Alpuche-Aviles, H. White, V. Brasiliense, C. Kranz, W. Schmickler, K. Stevenson, C. Jing, M. Edwards, Faraday Discuss. 2016, 193, 387-413.

[16] M. A. Edwards, D. A. Robinson, H. Ren, C. G. Cheyne, C. S. Tan, H. S. White, Faraday Discuss. 2018, 210, 9-28.

S. Goines, J. E. Dick, J. Electrochem. Soc. 2020, 167, 037505.

[18] J. Ustarroz, M. Kang, E. Bullions, P. R. Unwin, Chem. Sci. 2017, 8, 1841-1853.

[19] C. A. Little, R. Xie, C. Batchelor-Mcauley, E. Kätelhön, X. Li, N. P. Young, R. G. Compton, Phys. Chem. Chem. Phys. 2018, 20 , 13537-13546.

[20] K. Kanokkanchana, E. N. Saw, K. Tschulik, ChemElectroChem 2018, 5, 3000-3005.

[21] D. A. Robinson, M. A. Edwards, H. Ren, H. S. White, ChemElectroChem 2018, 5, 3059-3067.

[22] M. Brunet Cabré, D. Djekic, T. Romano, N. Hanna, J. Anders, K. McKelvey, ChemElectroChem 2020, 7, 4724-4729.

[23] R. Gao, M. A. Edwards, J. M. Harris, H. S. White, Curr. Opin. Electrochem. 2020, 22, 170-177.

[24] Y. Wang, X. Shan, N. Tao, Faraday Discuss. 2016, 193, 9-39.

[25] Y. Wang, Z. Cao, Q. Yang, W. Guo, B. Su, Anal. Chim. Acta 2019, 1074, 1-15.

[26] S. M. Lu, Y. Y. Peng, Y. L. Ying, Y. T. Long, Anal. Chem. 2020, 92, 5621-5644.

[27] S. E. Fosdick, K. N. Knust, K. Scida, R. M. Crooks, Angew. Chemie - Int. Ed. 2013, 52, 10438-10456.

[28] J. P. Guerrette, S. M. Oja, B. Zhang, Anal. Chem. 2012, 84, 16091616.

[29] J. P. Guerrette, S. J. Percival, B. Zhang, J. Am. Chem. Soc. 2013, 135, 855-861.

[30] S. M. Oja, J. P. Guerrette, M. R. David, B. Zhang, Anal. Chem. 2014 86, 6040-6048.

[31] W. Xu, C. Ma, P. W. Bohn, ChemElectroChem 2016, 3, 422-428.

[32] J. S. Stefano, F. Conzuelo, J. Masa, R. A. A. Munoz, W. Schuhmann, J. Electroanal. Chem. 2020, 872, 113921.

[33] Y. Fan, R. Hao, C. Han, B. Zhang, Anal. Chem. 2018, 90, 1383713841.

[34] R. Djoumer, A. Anne, A. Chovin, C. Demaille, C. Dejous, H. Hallil, J.

L. Lachaud, Anal. Chem. 2019, 91, 6775-6782.

[35] M. Quan, D. Sanchez, M. F. Wasylkiw, D. K. Smith, J. Am. Chem. Soc. 2007, 129, 12847-12856.

[36] X. Gao, J. Lee, H. S. White, Anal. Chem. 1995, 67, 1541-1545.

[37] N. Baltes, L. Thouin, C. Amatore, J. Heinze, Angew. Chemie - Int. Ed. 2004, 43, 1431-1435.

[38] C. Amatore, C. Pebay, L. Thouin, A. Wang, J. S. Warkocz, Anal. Chem. 2010, 82, 6933-6939.

[39] V. Sundaresan, K. Marchuk, Y. Yu, E. J. Titus, A. J. Wilson, C. M. Armstrong, B. Zhang, K. A. Willets, Anal. Chem. 2017, 89, 922-928.

[40] P. Lefrançois, V. S. R. Vajrala, I. B. Arredondo, B. Goudeau, T. Doneux, L. Bouffier, S. Arbault, Phys. Chem. Chem. Phys. 2016, 18, 25817-25822.

[41] B. Zhao, F. A. Summers, R. P. Mason, Free Radic. Biol. Med. 2012, 53, 1080-1087.

[42] C. Y. Liu, A. J. Bard, Anal. Chem. 2005, 77, 5339-5343

[43] S. W. Feldberg, M. A. Edwards, Anal. Chem. 2015, 87, 3778-3783.

[44] A. Oleinick, I. Svir, C. Amatore, Curr. Opin. Electrochem. 2019, 13, 33-39. 


\section{Entry for the Table of Contents}

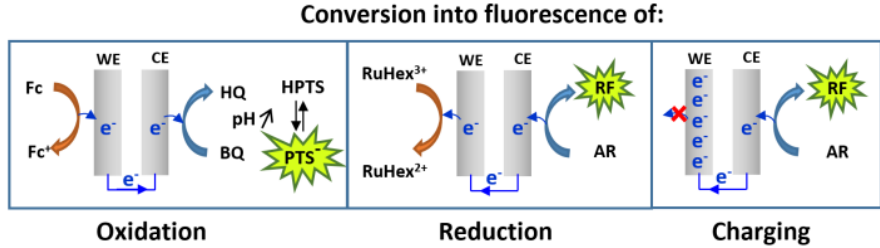

A universal potentiostatic platform for converting oxidative, reductive and even charging currents into fluorescence is described Performance improvements and limitations brought by using 8-hydroxypyrene-1,3,6-trisulfonic acid (HPTS) and Amplex Red as fluorogenic reporters are evidenced. Seldom reported coupling between a faradaic reaction and a capacitive charging process is directly visualized. 